\title{
Bilateral Diaphragmatic Paresis after Cardiac Surgery: The Key is the Clinical Point-of-Care Ultrasound
}

\author{
Jose Luis Vázquez Martínez ${ }^{1,2}$, Aida López de Pedro ${ }^{1 *}$, Ana Coca Pérez ${ }^{1}$, Raul Montero Yeboles ${ }^{1}$ and \\ Cesar Pérez-Caballero Macarrón ${ }^{1}$
}

${ }^{1}$ PICU, Hospital Universitario Ramón y Cajal, Madrid, Spain

${ }^{2}$ Department of Pediatric, Universidad Alcalá de Henares, Plaza de San Diego, Madrid, Spain

*Corresponding author: Aida López de Pedro, MD, PICU, Hospital Universitario Ramón y Cajal, Ctra. Colmenar Viejo, km. 9,100, 28034; Avda. Encuartes, $135^{\circ}$ A, 28760, Tres Cantos, Madrid, Spain, Tel: +34659284340, E-mail: aida.lopezdepedro@gmail.com

\begin{abstract}
Bilateral diaphragmatic paresis following cardiac surgery is rare. We present a case in which several extubation attempts failed in an infant after cardiac surgery. Point-of-care ultrasonography (POCUS) showed a slight but symmetric decrease of diaphragmatic motion, confirmed by fluoroscopy. Phrenic electromyography (EMG) was informed inconsistently as normal. After eight weeks of non-invasive ventilation the patient was weaned to room air, revealing the POCUS a recovery on the left side but persisting the right diaphragmatic hypomotility. Diagnosis, highly suspected by clinic and POCUS, resulted specially complicated because of the bilateral injury and the normal phrenic-EMG.
\end{abstract}

\section{Background}

Diaphragmatic paresis can be unilateral or bilateral $[1,2]$ and it is associated with prolonged mechanical ventilation and higher morbidity [3]. Phrenic nerve injury is relatively common after cardiac surgery (incidence 3-20\%) [1], being more frequent after Blalock-Taussig shunt surgery, Tetralogy of Fallot, Fontan procedure, and arterial switch operation [4]. Nerve injuries can be caused by local application of cold solutions for cardioplegia, excessive heat when using electric scalpel, nerve traction and, rarely, due to direct nerve section [1].

Clinical manifestations will depend on the uni- or bilateralism of the diaphragmatic affection, and the presence of underlying pulmonary disease. Bilateral diaphragmatic paresis (BDP) is less frequent, being usually symptomatic, and leading to respiratory failure despite the increase of the accessory muscles effort [2].

Several tools are available for the diagnosis of BDP but it is essential a high clinical suspicion. Both hemidiaphragms elevated with small lung volumes can be detected in chest X-ray (CXR) but this is difficult to assess [1]. Fluoroscopy, providing a real-time motion image, can show a decreased muscle excursion with paradoxical movement during inspiration, but implies ionizing radiation and the necessity to transfer the patient out of the Pediatric Intensive Care Unit (PICU). Overall when both hemidiaphragms are involved, diagnosis can be challenging due to the lack of normal hemidiaphragmatic motion on any side for comparison [1,2]. POCUS is a bedside, non-invasive, ionizing radiation-free tool potentially useful in the BDP diagnosis, by measuring the magnitude of diaphragmatic excursion, and its follow-up, by assessing the diaphragmatic recovery $[3,5]$. These features make it more attractive for young patients and those connected to mechanical ventilation. Phrenic-EMG exam can be also useful because it can detect evidence of denervation and also differentiate between neuropathic and myopathic causes of paralysis with high sensitivity and specificity and it can be performed in patients on full ventilator support. However, this technique has some limitations, mainly because of the wide range of values accepted as normal for children [6]. Usually the examination is considered normal when amplitude potentials for both hemidiaphragms are similar. 


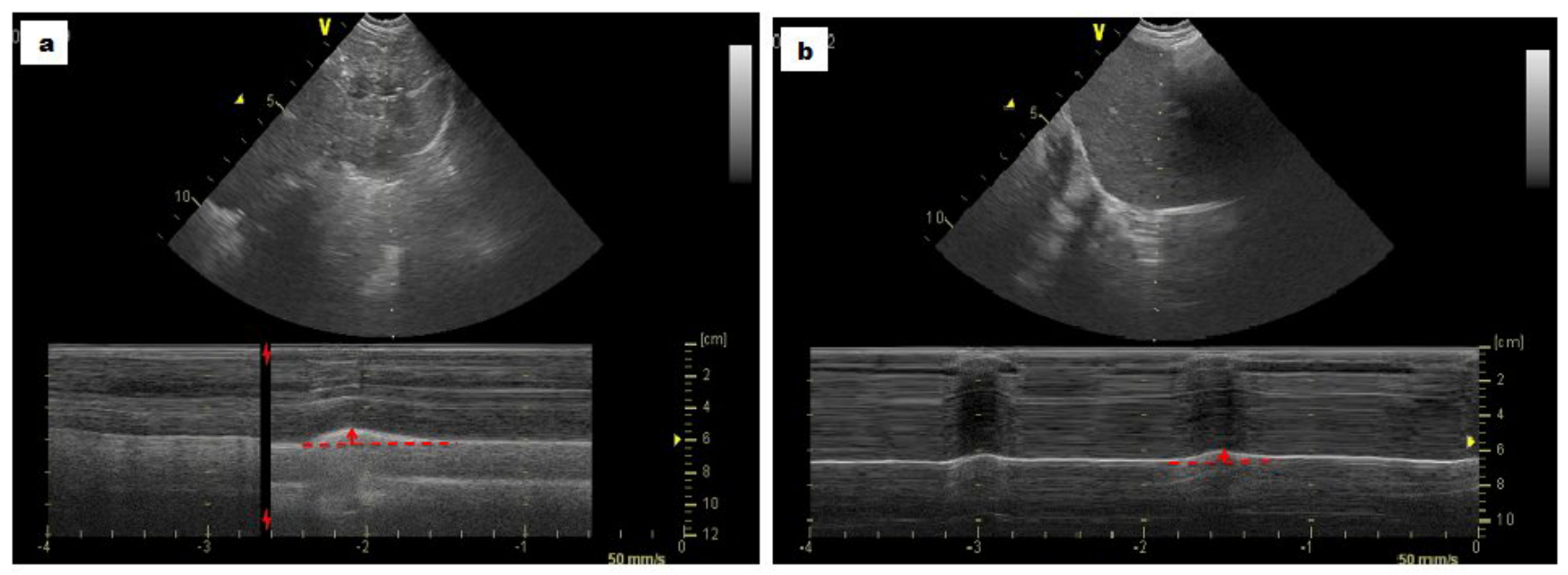

Figure 1: M-mode US a) Left best diaphragmatic excursion of $0.9 \mathrm{~cm}$; b) Right best diaphragmatic excursion of $0.7 \mathrm{~cm}$.

We report the case of an unusual BDP following cardiac surgery diagnosed by clinical and POCUS assessment despite other tests were inconclusive.

\section{Case Report}

A 4-month-old child was admitted to the PICU after a Ventricular Septal Defect was surgically repaired. The Unit admits all the critically ill children treated at the hospital, including those with cardiovascular diseases or after cardiac surgery. The surgery elapsed without remarkable incidences. After a planned extubation, the patient showed an immediate respiratory failure, requiring reintubation. She was mechanically ventilated for forty-eight additional hours when a new planned extubation failed, starting the child with respiratory distress and severe use of intercostal muscles, but without evidence of respiratory asymmetries. Non-invasive ventilation (NIV) was then started with clear clinical improvement. Echocardiography showed no ventricular dysfunction or residual defects and CXR showed clear lung fields with no diaphragm elevations.

Because of clinical suspicion of BDP, serial POCUS measurements were performed with a standard technique [3] by using a convex probe ( $4 \mathrm{C}$, VividT8, General Electrics ${ }^{\circledR}$ ). Bedside ultrasound showed basal bilateral B-lines without a consolidation pattern, positive but mild pleural sliding, and clearly decreased diaphragmatic motion on qualitative exam during quite breathing despite the best left and right diaphragmatic excursions during the deepest breathing were 0.9 centimeters (0.7$0.9 \mathrm{~cm}$ ) and $0.7 \mathrm{~cm}(0.55-0.7 \mathrm{~cm})$ respectively (Figure 1 ).

Even cold cardioplegia wasn't performed, our main suspicion was that the cause of the nerves palsy had been the exposure to low ambient temperatures at the operating room. There were no other causes that can better justify the patient's condition.

The third day after the surgery, fluoroscopy evidenced intermittent upward paradoxical movement but without asymmetries so the lack of a contralateral side to compare made the study inconclusive. Moreover, the lung expansion due to the intercostal muscles activity made the fluoroscopy much more difficult to interpret. A phrenic-EMG was performed but informed as normal probably due to the lack of references values of normality and/or the absence of differences between the values from both hemidiaphragms. After several weaning trial failures, a second fluoroscopy was performed the thirteenth day after surgery, showing anterior thorax excursion but clearly diminished in posterior regions, which reinforced the initial POCUS diagnosis of BDP. Patient was conservatively managed, supported by NIV for seven weeks when an important clinical improvement was observed. POCUS follow-ups were performed periodically without significant changes until the sixth week when a minimum of $0.7-0.9 \mathrm{~cm}$ of resting diaphragmatic excursion on the right side and $1.3-1.5 \mathrm{~cm}$ on the left side was observed. At this moment, a paradoxical diaphragmatic movement was also appreciated on the right side (Figure 2). Control CXR showed a right hemidiaphragm elevation and phrenic-EMG was asymmetric, showing an increase of motor distal latency of the right phrenic nerve, compared to the contralateral side. We suggest that it is due to a more severe affection of the right phrenic nerve.

\section{Comment}

We describe a case of BDP diagnosis particularly challenging due to the absence of surgical high risk factors, the presence of limited thoracic increase volume during the inspiration because of the use of accessory respiratory muscles, a first phrenic-EMG exam informed as normal and, above all, its bilateralism. Moreover, the diaphragmatic motion values measured by ultrasonography were in normal ranges according to some published data $[3,7]$. However, repetitive weaning failures plus abnormal respiratory dynamic and serial POCUS measurements showing poor diaphragmatic excursion during quiet breathing were essential for the diagnosis. Once the left side was recovered, the diagnosis of right paresis was definitive, and reinforced the initial diagnostic of post-operative BDP. The asymmetry between 


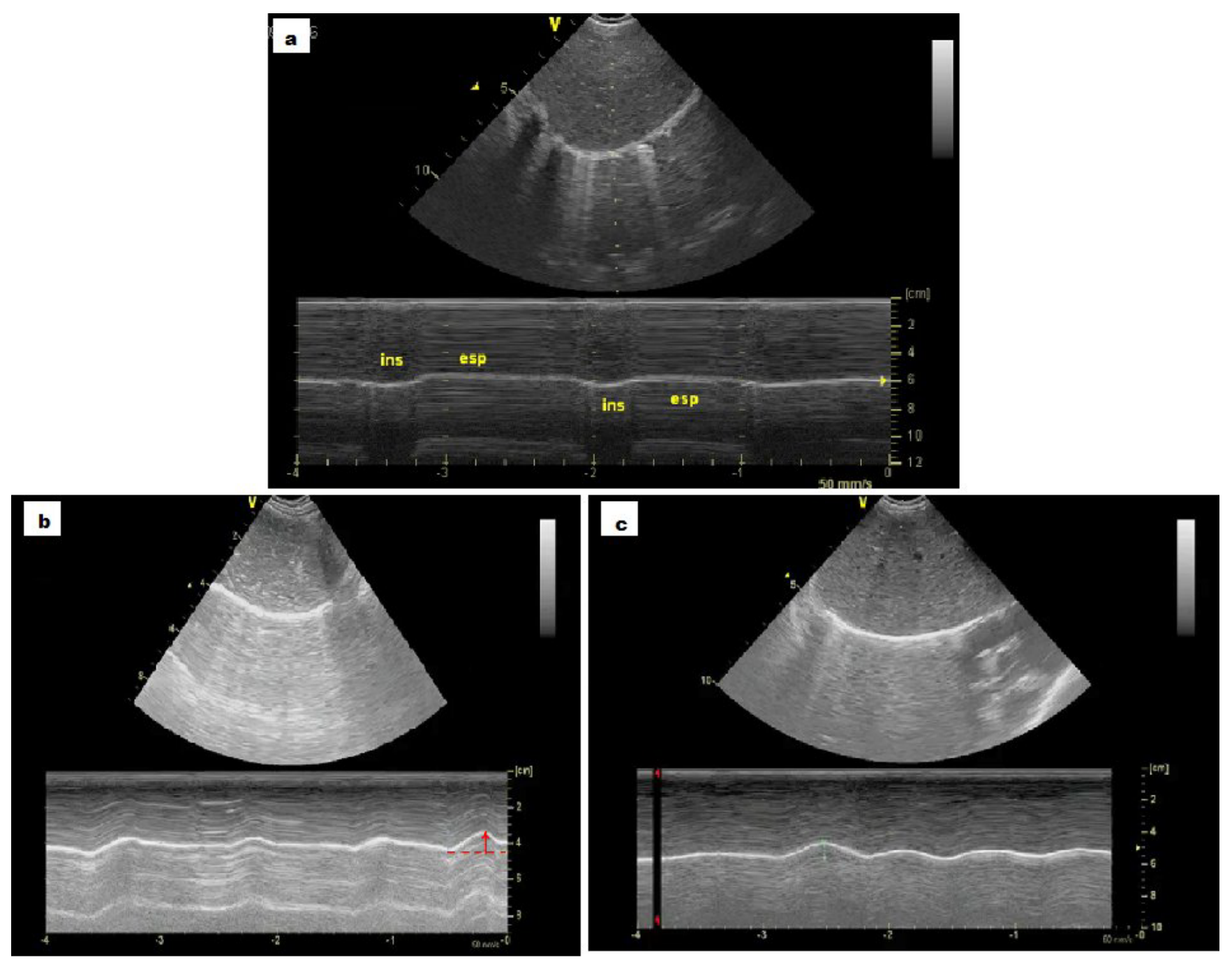

Figure 2: a) Paradoxical motion during inspiration on the right side; b) Three months after surgery, left side: Best diaphragmatic excursion $(1.3 \mathrm{~cm})$; c) Three months after surgery, right side: Best diaphragmatic excursion $(0.9 \mathrm{~cm})$.

both hemidiaphragms was finally also evidenced by a second phrenic-EMG evaluation.

Clinical suspicion of BDP must rise in those patients after cardiac surgery requiring prolonged ventilation, once other causes of respiratory weaning failure are excluded [1], as it happened in our patient. Unfortunately, the absence of asymmetry in diaphragmatic motility makes more difficult the clinical, radiological, ultrasonographic and electromyography evaluation [8]. Thus, BDP can be often missed in fluoroscopy examination because both hemidiaphragms move in concert [3].

Regarding the ultrasound, it could be limited by the absence of validated values for the diaphragmatic motion in children according to their age. Some authors consider normal when the diaphragmatic movement during inspiration towards the transducer is more than $4 \mathrm{~mm}$ or a difference between both hemidiaphragms is less than fifty percent $[3,7]$. These data turned out limited in our case.

As we showed in our patient (Figure 2a) and it has been published [4], the diaphragmatic paradoxical movement could be detected by POCUS by using the M-mode in a subcostal transverse view when checking both hemidiaphragms excursions. Instead of detecting a movement upwards (towards the probe) during inspiration, in patients affected by unilateral injury a downwards diaphragmatic excursion is seen during inspiration (Figure 3). In our opinion, a qualitative ultrasonographic assessment performed by skilled personnel improves the usefulness of ultrasound exam. It provides a continuous diaphragmatic assessment along the weaning process and gives a more realistic view of the diaphragmatic function than one single measurement as it is the best hemidiaphragm excursion. Although a patient could reach a normal single best excursion, it could be not enough for a successful weaning, which implies a continuous respiratory work.

Although the conservative management is the more appropriate treatment in most of the cases, before setting this approach, a correct diagnostic must be done, excluding other pathologies. So the important fact is to find an appropriate test to diagnose and follow-up the BDP when weaning failures occur.

In conclusion, bilateral diaphragmatic paresis diagnosis in infants requires high clinical index of suspicion and skilled POCUS evaluation. 


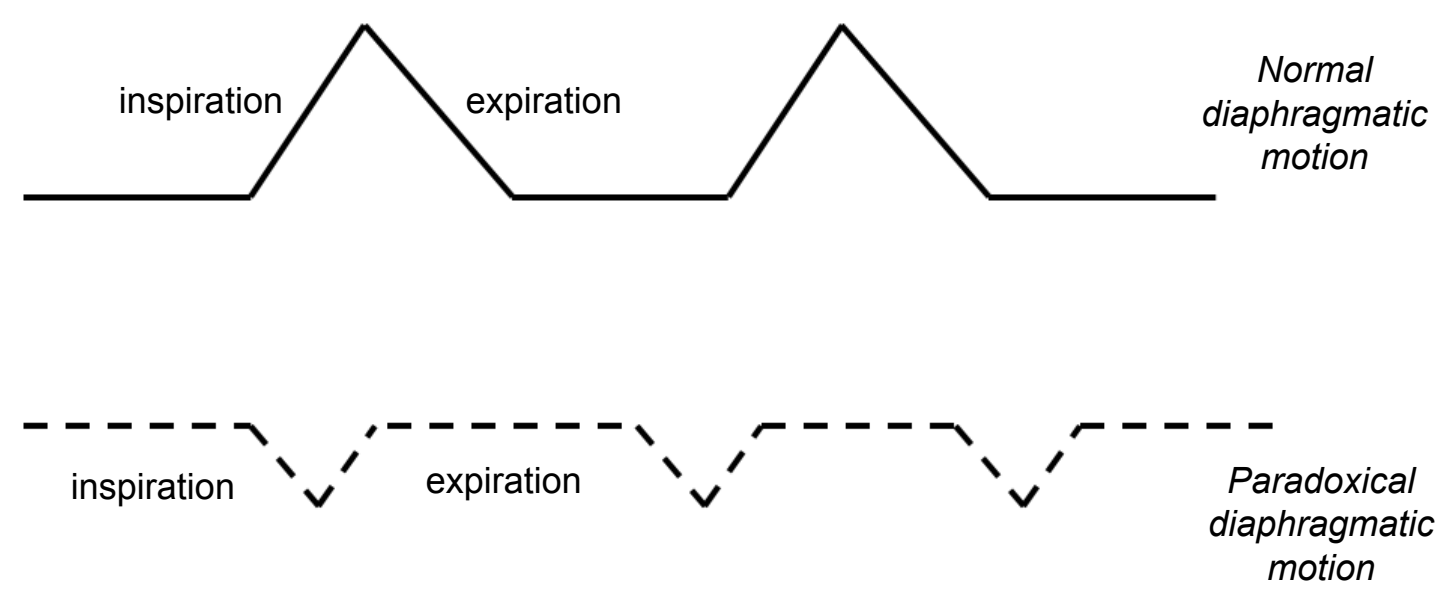

Figure 3: M-mode subcostal view: Normal diaphragm shows movement upwards (towards the probe) during inspiration while in the affected side movement downwards and decreased magnitude is noticed during inspiration.

\section{References}

1. Ko MA, Darling GE (2009) Acquired paralysis of the diaphragm. Thorac Surg Clin 19: 501-510.

2. Kharma N (2013) Dysfunction of the diaphragm: Imaging as a diagnostic tool. Curr Opin Pulm Med 19: 394-398.

3. Gil-Juanmiquel L, Gratacós M, Castilla-Fernández Y, Piqueras J, Baust T, et al. (2017) Bedside ultrasound for the diagnosis of abnormal diaphragmatic motion in children after heart surgery. Pediatr Crit Care Med 18: 159-164.

4. Sanchez de Toledo J, Munoz R, Landsittel D, Shiderly D, Yoshida M, et al. (2010) Diagnosis of abnormal diaphragm motion after cardiothoracic surgery: Ultrasound performed by a cardiac intensivist vs. fluoroscopy. Congenit Heart Dis 5: 565-572.
5. Urvoas E, Pariente D, Fausser C, Lipsich J, Taleb R, et al. (1994) Diaphragmatic paralysis in children: Diagnosis by TM-mode ultrasound. Pediatr Radiol 24: 564-568.

6. Sarwal A, Walker FO, Cartwright MS (2013) Neuromuscular ultrasound for evaluation of the diaphragm. Muscle Nerve $47: 319-329$.

7. El-Halaby $\mathrm{H}$, Abdel-Hady $\mathrm{H}$, Alsawah $\mathrm{G}$, Abdelrahman $\mathrm{A}$, El-Tahan H (2016) Sonographic evaluation of diaphragmatic excursion and thickness in healthy infants and children. J Ultrasound Med 35: 167-175.

8. Liet JM, Dejode JM, Joram N, Gaillard Le Roux B, Péréon Y (2013) Bedside diagnosis of bilateral diaphragmatic paralysis. Intensive Care Med 39: 335. 